\title{
Images
}

\section{Progressive bruising in an otherwise healthy child: immune thrombocytopenia purpura}

\section{Jason D. Heiner, MD*; Trent L. Morgan, $\mathrm{MD}^{\dagger}$}

A 3-year-old previously healthy female presented to the emergency department with 1 week of atraumatic bruising after having a viral upper respiratory infection. She appeared playful and was afebrile, with widespread petechiae, purpura, and ecchymoses on her skin and oropharynx (Figure 1). Her laboratory studies revealed a platelet count of $2 \times 10^{3} / \mu \mathrm{L}$ (normal 150 $400 \times 10^{3} / \mu \mathrm{L}$ ) with a normal white blood cell count, hemoglobin, and serum creatinine. A diagnosis of immune thrombocytopenia purpura (ITP) was made. She was given oral prednisone, and Pediatric Hematology was consulted for hospital admission, intravenous immunoglobulin (IVIG) treatment, and observation. She was discharged home 2 days later with a platelet count of $33 \times 10^{3} / \mu \mathrm{L}$.
Acute pediatric ITP usually follows an acute infection, with an estimated frequency of 50 per 1,000,000 children annually and peak prevalence between 2 and 4 years of age. ${ }^{1-3}$ Autoantibody-mediated platelet destruction and impaired platelet production contribute to the presenting petechiae, purpura, and mucous membrane or life-threatening bleeding. ${ }^{1-4}$ Spontaneous resolution generally occurs within 2 to 3 months. ${ }^{1,2,4,5}$ Interventions such as steroids, IVIG, or anti-D immunoglobulin can increase platelets to reduce the risk of life-threatening hemorrhage, which is most common with platelet counts below $10 \times 10^{3} / \mu \mathrm{L}^{1,2,4,5}$ In children, IVIG given alone may result in a greater acute rise in platelet count than seen with steroids. Platelet transfusion is reserved for severe hemorrhage. ${ }^{1-5}$ There are other potential causes of

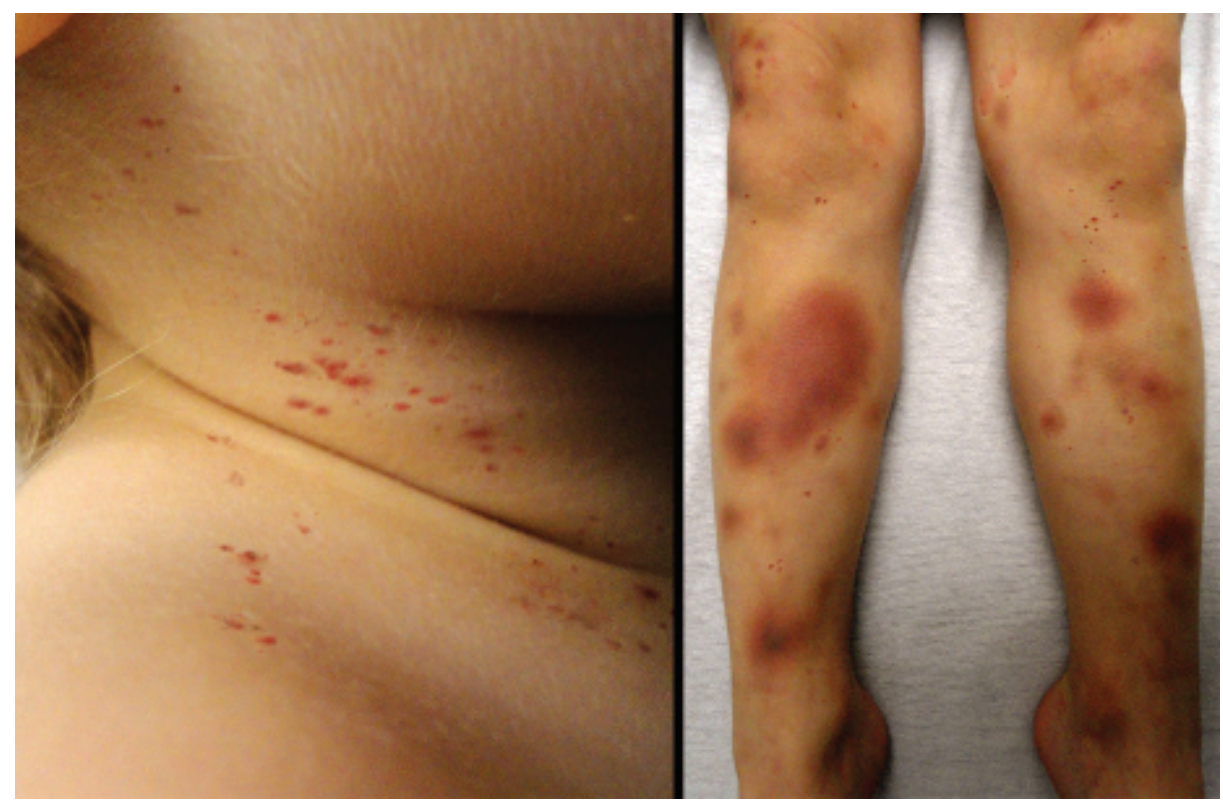

Figure 1. Scattered petechiae over the patient's face, neck, and upper torso (left) and appearance of the patient's lower extremities with diffuse petechiae, purpura, and regions of ecchymoses (right).

From the *Division of Emergency Medicine, University of Washington, Seattle, WA; †Department of Emergency Medicine, Brooke Army Medical Center, Fort Sam Houston, TX.

Correspondence to: Dr. Jason D. Heiner, Division of Emergency Medicine, University of Washington, 1959 Northeast Pacific Street, Box 356123, Seattle, WA 98195; jheiner@uw.edu.

This article has been peer reviewed. 
thrombocytopenia, such as drug interactions and inherited thrombocytopenias. In addition, some aspects of treatment remain controversial, so consultation with a pediatric hematologist should be considered. ${ }^{2}$

Competing interests: None declared.

Keywords: immune thrombocytopenic purpura, pediatrics

\section{REFERENCES}

1. Cines DB, Bussel JB, Liebman HA, et al. The ITP syndrome: pathogenic and clinical diversity. Blood 2009;113:6511-21, doi:10.1182/blood-2009-01-129155.
2. Provan D, Stasi R, Newland AC, et al. International consensus report on the investigation and management of primary immune thrombocytopenia. Blood 2010;115:168-86, doi:10.1182/ blood-2009-06-225565.

3. Stasi R. Pathophysiology and therapeutic options in primary immune thrombocytopenia. Blood Transfus 2011;9:262-73.

4. Zeller B, Rajantie J, Hedlund-Treutiger I, et al. Childhood idiopathic thrombocytopenic purpura in the Nordic countries: epidemiology and predictors of chronic disease. Acta Paediatr 2005;94:178-84, doi:10.1080/08035250410025294.

5. Beck CE, Nathan PC, Parkin PC, et al. Corticosteroids versus intravenous immune globulin for the treatment of acute immune thrombocytopenic purpura in children: a systematic review and meta-analysis of randomized controlled trials. 7 Pediatr 2005;147:521-7, doi:10.1016/j.jpeds.2005.04.032. 